\title{
Preclinical study of Doxorubicine-loaded liposomal drug delivery for the treatment of head and neck cancer: Optimization by Box-Behnken statistical design
}

\author{
Baihui Yang ${ }^{\square}$ \\ Department of Cardiovascular Surgery, The Second Affiliated Hospital of Kunming Medical University, Kunming, Yunnan, 650101, China
}

The present investigation aimed at developing Doxorubicin (DOX)-loaded liposome-mediated drug delivery system for head and neck cancer. The liposomes were prepared by film hydration technique using egg phosphatidylcholine and cholesterol using Box-Behnken statistical design. The prepared liposomes were evaluated for the percentage encapsulation efficiency, particle size and in vitro release. The average particle size of the DOX-encapsulating liposomes formulated by thin-film hydration technique was between $150.5 \mathrm{~nm}$ and $200 \mathrm{~nm}$ with an average particle size of $165.80 \mathrm{~nm}$. The PDI (Polydispersity index) was found to be $\mathbf{0 . 3 1 5}$ which indicated that particles were monodispersed and narrow-dispersed. In vitro drug release of DOX-loaded liposomes and DOXloaded peptide-conjugated liposomes was performed in phosphate buffered saline ( $\mathrm{pH} \mathrm{7.4)} \mathrm{and} \mathrm{both} \mathrm{formula-}$ tions showed sustained release behavior over the period of $\mathbf{4 0}$ hours. The optimized liposomal formulation was conjugated to a peptide and subsequently radiolabeled with ${ }^{186}$ Re-perrhenate solution and BMEDA-glucoheptonate-stannous chloride solution. Comparative cytotoxicity assay of DOX, DOX-liposomes and DOX-liposomespeptide on SCC9 cells was performed and it was found that liposomal formulation was not cytotoxic. The antitumor efficacy of ${ }^{186} \mathrm{Re}$-liposomes, unlabelled liposomes, ${ }^{186}$ Re-perrhenate solution and ${ }^{186} \mathrm{Re}-\mathrm{BMEDA}$ solution was determined in SCC cell lines injected into BALB/c-nu/nu athymic nude rats. The efficacy of antitumor activity was found to be in the following order: peptide-conjugated DOX-loaded liposomes> unlabelled liposomes $>186$ Re-perrhenate solution $>{ }^{186}$ Re-BMEDA solution. The present investigation showed that peptide-conjugated DOX-loaded liposomes significantly suppress the tumor growth in the nude rat model. These results suggest the significant potential of liposomes as carriers for clinical applications in head and neck cancer.

Key words: liposomes, head and neck, cancer, doxorubicine

Received: 22 December, 2019; revised: 01 April, 2020; accepted: 07 April, 2020; available on-line: 08 June, 2020

๑e-mail: kmyangbh@sina.com

Abbreviations: ATCC, American Type Culture Collection; BMEDA, $\mathrm{N}, \mathrm{N}$-bis (2-mercaptoethyl)-N',N'-diethylethylenediamine; $\mathrm{CH}$, Cholesterol; DOX, Doxorubicin; EE, Encapsulation efficiency; EPC, Egg phosphatidylcholine; HNC, Head and neck cancer; HSCC, Human squamous cell carcinomas; IT, Interventional Therapies; LITT, Laserinduced thermotherapy; MCT, Microwave coagulation therapy; PDI, Polydispersity inde; PEI, Percutaneous ethanol injection; SEM, Scanning electron microscopy; RFA, Radiofrequency ablation; TACE, Transarterial chemoembolization

\section{INTRODUCTION}

Head and neck cancer (HNC) is human squamous cell carcinomas (HSCC). It is a group of cancer comprising of tumors arising from upper aerodigestive tract to salivary glands and thyroids (mouth, nose, throat, larynx, sinuses, or salivary glands). HNC affects more than 30000 people yearly in the United States (Wikipedia/ head neck cancer). The condition can be diagnosed with typical symptoms like non-healing sore throat, trouble while swallowing, sometimes voice change and breathing problems. Unusual bleeding, facial swelling, or breathing difficulty can be observed in some symptomatic patients. The prime reason underlying the malignant condition (about $75 \%$ of all HNC cases) is chewing tobacco. Tissue biopsy is the only measure to confirm the disease, however, medical imaging and blood tests can be used to assess the disease spread. The preventive measures for HNC can be stated as avoidance of alcohol and tobacco. The disease is curable if detected at an early stage. Chemotherapy, use of radiation, targeted therapy are common treatment suggestions which may be used either alone or in combination with each other. After receiving treatment for $\mathrm{HNC}$, the patients become at high risk for other types of cancer (head neck cancer/NCI; WHO., World Cancer Report., 2014).

Apart from chemotherapy in non-surgical approach, regional Interventional Therapies (IT) were the breakthrough for successful handling of HNC (Qian et al., 2003). IT includes radiofrequency ablation (RFA), percutaneous ethanol injection (PEI), microwave coagulation therapy (MCT), laser-induced thermotherapy (LITT), transarterial chemoembolization (TACE) etc. These invasive drug delivery methods seem to be promising as a viable treatment option for human cancer (Poon et al., 2002). It is due to the fact of showing extended survival rates with minimal possible tissue and organ toxicity resulting in the improved level of quality life in cancerous patients. The reason underlying using these techniques in cancer therapy is the improved imaging methods allowing accurate and effective treatment application. Interventional radiology is a medical tool which uses radiology for invasive image-guided procedures to treat diseases in humans. The idea behind interventional radiology is to treat cancer patients using the least invasive techniques with minimal risk to their lives and improve the health outcomes. These procedures demonstrated less risk, less pain and shorter recovery time when compared to the open surgery (Harrington et al., 2001).

Colloidal liposomes emerged as potential drug delivery system which uses microscopic and submicroscopic vesicles containing biocompatible, biodegradable and 
Table 1. Different levels of independent and dependent variables

\begin{tabular}{llll}
\hline Independent Variables & Low level $(-1)$ & Medium level $(0)$ & High level $(+1)$ \\
\hline A, EPC (M) & 2 & 2.5 & 3 \\
\hline B, Cholesterol (M) & 0.8 & 1.0 & 1.2 \\
\hline C, Chloroform $(\mathrm{ml})$ & 150 & 175 & 200 \\
\hline
\end{tabular}

Dependent Variables

$\mathrm{Y} 1, \% \mathrm{EE}$

non-toxic composite (Bao et al., 2006). Liposomes are potential drug delivery system for chemo, gene and radionuclide therapies. Liposomal diameter ranges from 10 $\mathrm{nm}$ to $20 \mu \mathrm{m}$ and they contain aqueous compartments enclosed by one or multiple lipid bilayers. Liposomal drug delivery system is non-toxic and biodegradable as they are formulated from phospholipids and their derivatives. A significant breakthrough was achieved in liposomal formulations for drug delivery applications and few liposomal drugs that are antifungal and antitumor were approved for clinical use (Elser et al., 2007).

The most important limitation of the chemotherapy is the unpredictable distribution of the cytotoxic chemotherapeutic agents to the body leading to the dose-limiting toxicity. To solve this problem, the drug molecule must be made available to the cancerous cells without affecting the other non-targeted areas (Herbst et al., 2005). Hsp47/CBP2 is an intracellular molecular chaperone found in the ER-Golgi. It was found that this protein leaks from the cancerous cells of the head and neck and is located on the cell surface. This creates an ideal situation for targeted delivery of anticancer drugs. In this study, we developed DOX-loaded peptide-conjugated liposomes for interventional therapy for the treatment of HNC.

\section{MATERIALS AND METHODS}

Doxorubicin (DOX) was obtained as a kind gift sample from Shouguang Fukang Pharmacy Factory (Shandong, China). Egg phosphatidylcholine (EPC) and dipalmitoylphosphatidylcholine, cholesterol $(\mathrm{CH})$ were kindly gifted by Lipoid GmbH, Ludwigshafen Germany. The Hsp47/CBP2 targeting peptide was obtained from Sigma Genosys USA.

Formulation of DOX-enclosing liposomes by thin-film hydration method (Cohen et al., 2010). OXloaded liposomes were formulated using EPC and $\mathrm{CH}$ in various equimolar ratios with $\mathrm{SA}$ comprising about $5 \%$ of the total lipids. Liposomes were prepared by conventional thin-film hydration method. Approximately $7 \mathrm{ml}$ of chloroform in $50 \mathrm{ml}$ round bottom flask was used to dissolve the lipids. To this lipidic solution $100 \mathrm{mg}$ of DOX dissolved in $5 \mathrm{ml}$ of methanol was added under constant stirring. Rotary evaporator (Heidolph) was used to remove the organic solvent phase like chloroform and methanol, leaving behind thin lipid film on the side wall of the flask which was subsequently dried using nitrogen for nearly 3 hours and the trace amounts of organic phases were completely removed. The dried film was hydrated with $15 \mathrm{ml}$ saline phosphate buffer solution having $\mathrm{pH}$ 7.4. This hydrated lipidic suspension was shaken mechanically for 1 hour to form multilamellar liposomes. The lipidic suspension was centrifuged at 40000 RPM for 2 hours at $-7^{\circ} \mathrm{C}$ using a cooled centrifuge to separate drug-loaded liposomes from unentrapped DOX. The liposomal suspension was freeze-dried with the addition of trehalose as cryoprotectant at $-10^{\circ} \mathrm{C}$ under vaccum for 50 hours. The free-flowing powder or suspension was used for further analysis.

Design of the experiments. The statistical design of Box-Behnken was used for the development and optimization of the DOX liposomes. EPC (A), cholesterol (B) and chloroform (C) were chosen as independent variables and perecentage EE (Encapsulation efficiency) (A) was selected as the dependent variable. A polynomial equation of second order and contour plots were plotted to generate the responses. Different levels of the dependent variables were shown in Table 1.

DOX-loaded and peptide-conjugated liposomes preparation. The conjugation of the peptide was performed on DOX-loaded liposomes as described by Liang Cheng (Cheng et al., 2014). Briefly, the peptide was covalently bound to the liposomes (of optimized formulation) by aminolysis. To the suspension of DOX-loaded liposomes, 1.2 M peptide solution in saline phosphate buffer $\mathrm{pH} 7.2$ was added. The solution was kept under stirring in the dark at room temperature for a day. DOX-loaded peptide-conjugated liposomes were separated from the mixture by ultracentrifugation at $-80^{\circ} \mathrm{C}$. The precipitated conjugate was dispersed in double-distilled water followed by lyophilization to obtain free-flowing liposomal conjugate system (Cheng et al., 2014).

Radiolabeling of the DOX-loaded peptide-conjugated liposomes. Radiolabeling of the optimized liposomes was performed by a previously reported method. Radiolabeling was performed by adding the ${ }^{186}$ Re-perrhenate solution to BMEDA (N,N-bis (2-mercaptoethyl)N',N'-diethylethylenediamine)-glucoheptonate-stannous chloride solution and incubating at $80^{\circ} \mathrm{C}$ for 1 hour. BMEDA-glucoheptonate-stannous chloride solution was prepared by dissolving $1.34 \mathrm{mg}$ BMEDA, $22.3 \mathrm{mg}$ glucoheptonate and $1.6 \mathrm{mg}$ stannous chloride in $\mathrm{pH} 5.0$ saline solution. After incubation, ${ }^{186}$ Re-BMEDA solution was cooled at room temperature with $\mathrm{pH}$ equivalent to 7.0. To this solution $2.0 \mathrm{ml}$ of the liposomal solution was added containing $4.44 \mathrm{GBq}{ }^{186}$ Re-activity and incubated for $1 \mathrm{~h}$ at $37^{\circ} \mathrm{C}$. Labeled ${ }^{186}$ Re-liposomes were separated from free ${ }^{186}$ Re using PD-10 columns (Farokhzad et al., 2009).

Intratumoral Drug Infusion and Image Acquisition. Human oral SCC cell lines (SCC4 and SCC9) were obtained from American Type Culture Collection (ATCC). Cell culture medium was purchased from Himedia, Shanghi China. The head and neck SCC xenografts model was designed as reported by $\mathrm{Bao} \mathrm{A}$ et al (Bao et al., 2006). In brief, $5 \times 10^{6}$ SCC4 cells were inoculated to each male $r m$ / rnu athymic nude healthy rat of 5-6 weeks age weighing 80-110 g. All animal experiments were performed according to the rules and regulations of the animal ethical committee of Department 
of Cardiovascular Surgery, The Second Affiliated Hospital of Kunming Medical University, Kunming, Yunnan, 650101, China. The animals were under observation during the whole study to detect tumor growth. The tumor weight, size, volume, length, width were measured when tumor became palpable. When tumor volume reached $2.5 \mathrm{~cm}^{3}$, therapy with doxorubicin-loaded and peptideconjugated liposomes was applied by convection-enhanced intratumoral administration. To understand the potential role of liposomes as a drug carrier for the interventional cancer therapy, the tumor-bearing nude rats were divided into 4 groups (24 nude rats in total). One group was treated with ${ }^{186} \mathrm{Re}$-liposomes and the remaining 3 groups were considered as control groups. The three control groups were injected with 1) unlabelled liposomes 2) ${ }^{186} \mathrm{Re}$-perrhenate solution 3) ${ }^{186} \mathrm{Re}-\mathrm{BMEDA}$ solution.

\section{Characterization of DOX-loaded liposomes and DOX- loaded peptide-conjugated liposomes}

Particle size and morphology. The freeze-dried liposomes were suspended in double-distilled water and sonicated for $1 \mathrm{~min}$ before analysis. Particle size, particle size distribution and zeta potentials were measured using a Zetasizer (Malvern instruments; DTS Ver 4.10) at room temperature. Topical morphology was examined by scanning electron microscopy (SEM) (Leo 435 VP, Cambridge, UK). For zeta potential determination the working distance of $8.5-8.7 \mathrm{~mm}$ was maintained with an accelerating voltage of approximately $15.0 \mathrm{kV}$. The liposomes had to be made electrically conductive by coating them with gold. The gold-coated liposomes were placed firmly on a brass tub using double-sided adhesive tape. During the entire procedure, a vacuum (5 pa) in an ion sputter (Hitachi E1010) was maintained.

Drug encapsulation efficiency (EE). The supernatant obtained after centrifugation was used for determining non-encapsulated DOX by HPLC (Young lin, Germany). The supernatant $(20 \mu \mathrm{l})$ was injected into a chromatograph equipped with a UV detector and C18 column. The mobile phase that was found suitable was acetonitrile/HPLC grade water $(85 \%: 15 \%, \mathrm{v} / \mathrm{v}$, with $0.16 \%$ $(\mathrm{w} / \mathrm{v})$ trimethylamine and $0.16 \%(\mathrm{w} / \mathrm{v})$ ortho-phosphoric acid; flow rate $1.5 \mathrm{ml} / \mathrm{min}$, wavelength $240 \mathrm{~nm}$ ). EE of the liposomes was determined according to the following equation (Elser et al., 2007).

$$
\mathrm{EE}(\%)=(\text { Total drug }- \text { free drug } / \text { Total drug }) \times 100
$$

In vitro drug release study. A dialysis $\mathrm{bag} / \mathrm{mem}-$ brane (molecular cut-off at $5 \mathrm{kDa}$ ) method was used to determine DOX release from the liposomes. The equivalent of $20 \mathrm{mg}$ DOX-loaded liposomes was dispersed in

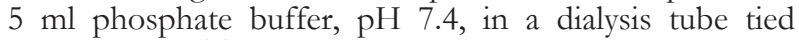
at both ends. The tube was placed in $200 \mathrm{ml} \mathrm{PBS} \mathrm{solu-}$ tion in a beaker. The system was maintained at $37^{\circ} \mathrm{C}$ and kept under stirring by means of a magnetic stirrer. At predetermined time points, $5 \mathrm{ml}$ of the solution was removed and replaced with a fresh solution so as to maintain sink conditions. DOX release at each time point was measured by the HPLC method as described above (Chung et al., 2004).

Cell culture and cytotoxic assay. A cytotoxic assay was performed on the SCC9 cells, as described by Dietz and others (Dietz et al., 2010). The cell line was cultured in Dulbecco's Modified Eagle Medium containing fetal bovine serum $(10 \%, \mathrm{v} / \mathrm{v})$, penicillin $(100 \mathrm{UI} / \mathrm{ml})$ and streptomycin sulfate $(100 \mu \mathrm{g} / \mathrm{mL}, \mathrm{pH} 7.2-7.4)$. A slightly humid environment with $5 \%(\mathrm{v} / \mathrm{v}) \mathrm{CO}_{2}$ was main- tained with a temperature of $37^{\circ} \mathrm{C}$. The cultured cells were seeded onto 48 -well tissue culture plates $\left(2 \times 10^{4}\right.$ cells/well) 48 hours before the experiment. These cells were incubated for $24 \mathrm{~h}$ with test compounds (DOXliposomes, DOX-lipo-peptide and blank-lipo). The test compounds were used at two different concentrations $(0.25$ and $0.50 \mathrm{mg} / \mathrm{ml})$. The MT'T assay was used to assess the cell viability. In this assay, MT'T $(1 \mathrm{mg} / \mathrm{mL})$ was incubated with test compound-treated cancer cell lines for $1 \mathrm{~h}$ at $37^{\circ} \mathrm{C}$. Cell viability was assessed from the amount of MTT transformed into insoluble formazan. This insoluble crystal form was made soluble in a $1 \mathrm{M}$ $\mathrm{HCl}$ /isopropyl alcohol mixture (1:24, v/v) and shaken for $20 \mathrm{~min}$ at room temperature.

\section{RESULTS AND DISCUSSION:}

\section{Statistical analysis of DOX encapsulation efficiency (EE)}

Human squamous cell carcinoma of the head and neck is characterized by over-expression of a tumor cell surface-specific receptor named Hsp47/CBP2 which makes it a favorable candidate for targeted delivery of anticancer drugs. The purpose of this study was to investigate the potential of liposome-doxorubicin (Dox)peptide conjugates containing a Hsp47/CBP2 binding peptide sequence, namely WHYPWFQNWAMA, for targeted delivery. For this, the liposomal formulation was developed by the design of experiments (DOE). Behnken is 3-variable and 3-level statistical design containing independent variables $(\mathrm{A}, \mathrm{B}, \mathrm{C})$ at 3 levels $(-1$, $0,+1)$ used to study the effect on the dependent variables (Y1, Y2). From the results presented in Table 2 it was confirmed that DOX encapsulation efficiency varied with the independent variables (A: EPC, B: Cholesterol and C: Chloroform). It is generally accepted that percentage entrapment efficiency and drug loading increases with the cholesterol content. Similar results were obtained for the encapsulation efficiency of DOX. The percentage entrapment efficiency of DOX in liposomes composed of EPC alone was $20.25 \%$, whereas the entrapment efficiency increased to $78.89 \%$ in liposomes prepared with EPC and cholesterol. The further increase in the entrapment efficiency to $86.76 \%$ was observed for the liposomes composed of EPC, cholesterol and chloroform at the high level $(+1)$ of all independent variables. The drug loading of the DOX was also determined using Sephadex G-50 mini column. It was found that the drug loading was also increased with increasing EPC concentration. The drug loading was found between 7.5 and $12.50 \%$.

The efficiency of DOX encapsulation in liposomes was between $47.90 \%$ and $82.17 \%$. Transformed values for all liposomal formulations were presented in $\mathrm{Ta}$ ble 3. Predicted and observed values of the encapsulation efficiency were shown in Table 4. The encapsulation efficiency (Y1) was given by the following polynomial equation of second order:

$\mathrm{Y} 1=+71.71+4.61 \mathrm{~A}+13.53 \mathrm{~B}+1.51 \mathrm{C}-3.01 \mathrm{~A}^{2}-1.37 \mathrm{~B}^{2}-$

$2.90 \mathrm{C}^{2}+0.30 \mathrm{AB}-0.31 \mathrm{AC}+0.21 \mathrm{BC}$

A positive and negative value in the above regression equation showed a synergistic and antagonistic effect, respectively, between the factor and response (Raghavan et al., 2006). The correlation coefficient $\left(\mathrm{R}^{2}\right)$ was 0.9816 which indicates a good fit of the model as shown in Table 4. A wide variation was observed in EE of DOX from $30.45 \%$ to $86.78 \%$. This variation confirmed that 
Table 2. Formulation of batches.

\begin{tabular}{|c|c|c|c|c|}
\hline \multirow{2}{*}{ Batches } & \multicolumn{3}{|c|}{ Factor } & \multirow{2}{*}{$\begin{array}{l}\text { Resopnse } \\
\mathrm{Y} 1\end{array}$} \\
\hline & A & B & $C$ & \\
\hline F1 & 0 & 0 & 0 & 78.90 \\
\hline F2 & +1 & +1 & 0 & 72.71 \\
\hline F3 & 0 & -1 & -1 & 80.12 \\
\hline F4 & -1 & +1 & 0 & 82.17 \\
\hline F5 & -1 & 0 & -1 & 55.70 \\
\hline F6 & 0 & -1 & +1 & 75.80 \\
\hline F7 & 0 & +1 & +1 & 77.98 \\
\hline F8 & 0 & 0 & -1 & 52.18 \\
\hline F9 & -1 & 0 & +1 & 47.90 \\
\hline F10 & -1 & -1 & 0 & 53.80 \\
\hline F11 & +1 & -1 & 0 & 78.45 \\
\hline F12 & 0 & 0 & 0 & 72.90 \\
\hline F13 & 0 & 0 & 0 & 72.90 \\
\hline F14 & 0 & +1 & -1 & 78.60 \\
\hline F15 & +1 & 0 & +1 & 70.82 \\
\hline F16 & 0 & 0 & 0 & 72.90 \\
\hline F17 & +1 & 0 & -1 & 68.87 \\
\hline
\end{tabular}

the EE was closely influenced by independent variables $(\mathrm{A}, \mathrm{B}, \mathrm{C})$. In the above regression equation $\mathrm{A}, \mathrm{B}$ and $\mathrm{C}$ represent the average result when 1 variable is changed at a time from lower to higher level. The terms AB, BC, $\mathrm{AC}$ represent the change in $\mathrm{EE}$ when two independent variables are simultaneously changed. The negative factors of the independent variables in the regression equation indicate the unfavorable effect on the EE while positive coefficients represent the favorable effect. As presented in Table 5 the model F-value of 41.41 shows the model was significant. Values of Prob $>$ F less than
0.0500 indicate that model terms were significant. In this statistical design $\mathrm{A}, \mathrm{B}, \mathrm{A}^{2}, \mathrm{C}^{2}$ were significant model terms $(p<0.0500)$. The $\mathrm{p}$ values greater than 0.1000 indicated that the model terms were not significant.

The combined effects of independent variables $A$ and $\mathrm{B}$ on EE of DOX were presented in Fig. 1A. It could be concluded from the contour plot that the EE increased with increasing concentration of EPC and cholesterol. The maximum EE of $86.76 \%$ was observed when both $A$ and $B$ were at a high level $(+1)$ in Run 2. The minimum EE of 48.51 was observed when both $A$ and $B$

Table 3. Diagnostics case statistics for various response variables.

\begin{tabular}{|c|c|c|c|c|}
\hline Batch No & Response Variables & Actual value & Predicted Value & Residual \\
\hline F1 & Y1 & 78.90 & 80.27 & -1.37 \\
\hline F2 & Y1 & 72.71 & 73.87 & -1.16 \\
\hline F3 & Y1 & 80.12 & 78.82 & 1.3 \\
\hline F4 & Y1 & 82.17 & 81.90 & 0.27 \\
\hline F5 & Y1 & 55.70 & 57.45 & -1.75 \\
\hline F6 & Y1 & 75.80 & 78.20 & -2.4 \\
\hline F7 & Y1 & 77.98 & 80.23 & -2.25 \\
\hline F8 & Y1 & 52.18 & 57.34 & -5.16 \\
\hline F9 & Y1 & 47.90 & 51.67 & -3.77 \\
\hline F10 & Y1 & 53.80 & 55.87 & -2.07 \\
\hline F11 & Y1 & 78.45 & 82.56 & -4.11 \\
\hline F12 & Y1 & 72.90 & 74.35 & -1.45 \\
\hline F13 & Y1 & 72.90 & 74.80 & -1.9 \\
\hline F14 & Y1 & 78.60 & 80.45 & -1.85 \\
\hline F15 & Y1 & 70.82 & 72.45 & -1.63 \\
\hline F16 & Y1 & 72.90 & 69.72 & 3.18 \\
\hline F17 & Y1 & 68.87 & 66.75 & 2.12 \\
\hline
\end{tabular}


Table 4. Summary of the results of regression analysis for responses $Y 1$

\begin{tabular}{llccccc}
\hline Models & $\mathrm{R}^{2}$ & Adjusted $\mathrm{R}^{2}$ & Predicted $\mathrm{R}^{2}$ & Std. Dev & Press & Remarks \\
\hline Response $\mathrm{Y} 1$ & & & & & & \\
\hline Linear & 0.9215 & 0.9167 & 0.9225 & 3.10 & 190.80 & $\ldots \ldots$ \\
\hline 2FI & 0.9420 & 0.9025 & 0.8712 & 3.45 & 210.27 & $\ldots \ldots \ldots$ \\
\hline Quadratic & 0.9967 & 0.9687 & 0.94867 & 1.16 & 150.89 & Suggested \\
\hdashline Cubic & 0.9730 & 0.9425 & 0.9198 & 2.55 & 110.25 & Aliased \\
\hline
\end{tabular}

Regression equation of the fitted models: $\mathrm{Y} 1=+71.71+4.61 \mathrm{~A}+13.53 \mathrm{~B}+1.51 \mathrm{C}-3.01 \mathrm{~A} 2-1.37 \mathrm{~B} 2-2.90 \mathrm{C} 2+0.30 \mathrm{AB}-0.31 \mathrm{AC}+0.21 \mathrm{BC}$

Table 5. ANOVA of models for $\mathrm{Y} 1$

\begin{tabular}{llllll}
\hline Source & DF & Sum of squares & Mean square & F value & P value \\
\hline Model for $\mathrm{Y1}$ & & & & & \\
\hline A & 1 & 169.92 & 169.92 & 16.79 & 0.0011 \\
\hline B & 1 & 1463.95 & 1463.95 & 144.68 & $<0.0001$ \\
\hline Residual & 14 & 141.66 & 10.12 & 11.33 & 0.3445 \\
\hline Lack of fit & 10 & 113.33 & 7.08 & 1.60 & \\
\hline Pure error & 4 & 28.32 & & & \\
\hline Core total Model 2 & 16 & 1775.53 & & \\
\hline
\end{tabular}

were at a low level (-1) in Run 10. So, EE was increased from $48.51 \%$ to $86.76 \%$. This increase in entrapment efficiency of DOX is explained by the fact that when the level of the cholesterol increases in lipidic bilayer the rigidity of the liposomes increases sharply showing higher stability and reduced permeability of the lipoidal membrane with greater retention. The combined effect of independent variables $\mathrm{A}$ and $\mathrm{C}$ on $\mathrm{EE}$ of DOX was shown in Fig. 1B. It was confirmed that with increasing concentration of EPC and chloroform, the EE of DOX also increased. Figure $1 \mathrm{C}$ explains the combined effect of $\mathrm{B}$ and $\mathrm{C}$ on the EE of DOX. Direct relationship was found between the concentration of $\mathrm{B}$ and $\mathrm{C}$ on the $\mathrm{EE}$ of DOX.
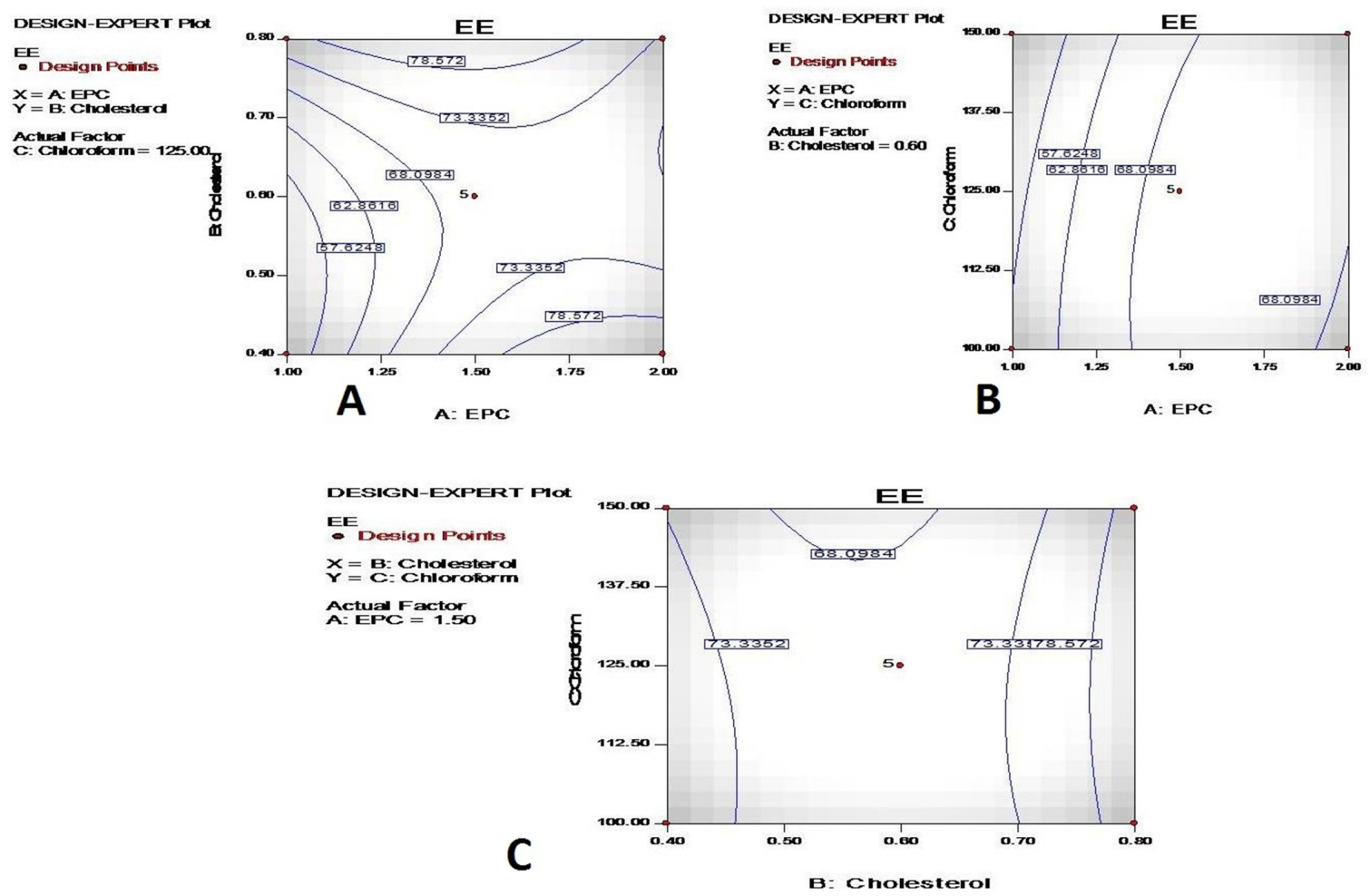

Figure 1. Contour plots.

(A) Effect of EPC and cholesterol on EE; (B) Effect of chloroform and EPC on EE; (C) Effect of cholesterol and chloroform on EE. 


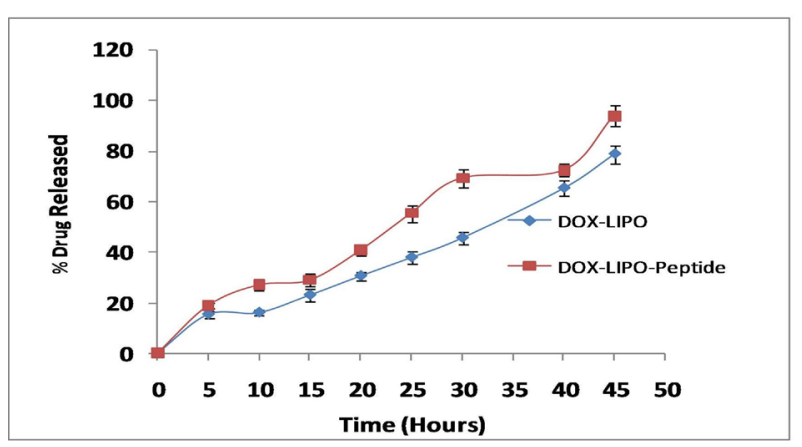

Figure 2. In-vitro drug release of DOX-loaded liposomes and DOX-loaded peptide-conjugated liposomes in phosphate buffered saline $(\mathrm{pH} 7.4)$.

\section{Drug release in vitro}

DOX release from the optimized formulation (F4) was studied in the saline solution of phosphate buffer $(\mathrm{pH}$ 7.4). The optimized formulation (F4) showed an initial burst of the release of $12.45 \%$ of the total DOX from the liposomes followed by a slower gradual release of the drug $(\sim 77.89 \%)$ towards the end of the $40-\mathrm{h}$ study period. However, DOX-loaded peptide-conjugated liposomes prepared using the optimized formulation (F4) also showed a sustained drug release pattern $(\sim 77.89 \%)$, as shown in Fig. 2. This pattern clearly indicated the tendency of the sustained drug release from the liposomes, which could be essential for cancer treatment.

\section{Particle size and morphology}

Optimal size of liposomes is the major prerequisite for intratumoral distribution and cellular internalization. A smaller particle size increases its area, leading to maximum access to the tumor region of head and neck. In the present study, a Malvern particle size analyzer (DVS) showed nanosized particles with a very narrow PDI (Polydispersity index). DOX-loaded liposomes had a diameter of $92.50 \mathrm{~nm}$ (F4) to $482.50 \mathrm{~nm}$ (F7) with PDI values from 0.120 to 0.384 . Zeta potentials of liposomal suspension were found to be between 28.50 and 53.57 $\mathrm{mV}$. Zeta potentials increased upon the incorporation of DOX into the liposomes.

\section{Cell culture and cytotoxic assay}

A cytotoxic assay was performed on the SCC9 cells (the MTT assay) in vitro. In this assay pure DOX, liposomal DOX and DOX with peptide-conjugated liposomes were used to treat the SCC9 cells during 24-h incubation. As presented in the dose-response bar graph (Fig. 3), all test formulations showed a direct relation-

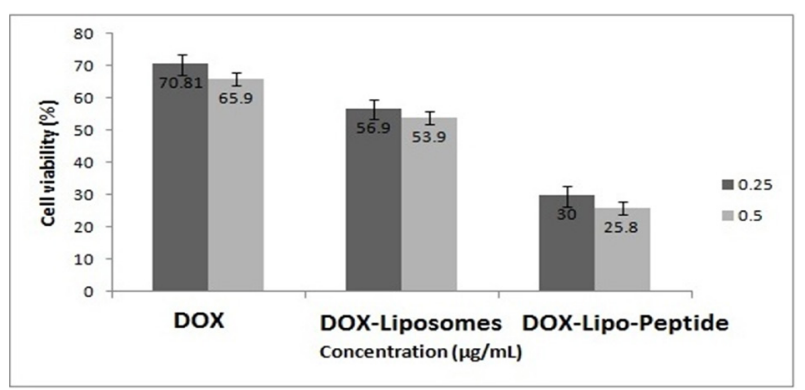

Figure 3. Comparative cytotoxic assay of DOX, DOX-liposomes and DOX-liposomes-peptide in SCC9 cells.

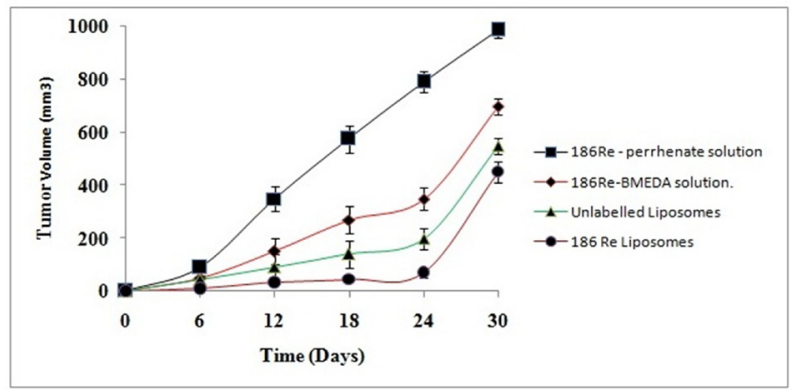

Figure 4. Comparison of antitumor efficacy in terms of tumor volume of ${ }^{186} \mathrm{Re}$-liposomes, non-conjugated liposomes, ${ }^{186} \mathrm{Re}-$ perrhenate solution and ${ }^{186}$ Re-BMEDA solution determined in SCC cell lines introduced to BALB/c-nu/nu athymic nude rats.

ship between the concentration used and cytotoxicity in SCC9 cells. The antiproliferative effect of DOX-loaded peptide-conjugated liposomes was found to be greater than that of DOX-loaded liposomes and pure DOX. The enhancement in the antiproliferative effect of DOXloaded peptide-conjugated liposomes could be attributed to the enhanced cellular uptake. The liposomes are thought to be initially captured by endocytic cells and then escape to reach acidic lysosomes from where the drug is continuously released in a gradual and sustained manner, leading to diffusion into the nuclear complex (Haddad et al., 2009).

\section{In vivo targeting assay}

Head and neck cancers are mainly found in squamous cell carcinomas which are characterized by gradual local expansion, invasion of nearer tissues and regional lymph node metastasis. In this research, a liposomal drug delivery technique was chosen to treat human head and neck SCC xenografts in nude rats which allowed the liposomes to disperse through the tumor interstitial spaces. ${ }^{186}$ Re-liposomes and remaining 3 groups were considered as control groups. The three control groups were injected with 1) unlabelled liposomes 2) ${ }^{186}$ Re-perrhenate solution 3) ${ }^{186}$ Re-BMEDA solution. The antitumor efficacy of ${ }^{186}$ Re-liposomes, unlabelled liposomes, ${ }^{186} \mathrm{Re}-$ perrhenate solution and ${ }^{186}$ Re-BMEDA solution was determined in vivo in SCC cells introduced to BALB/c-nu/ nu athymic nude rats. As shown in Fig. 4 the tumors in the untreated control group showed mean tumor volume of $850 \mathrm{~mm}^{3}$. In contrast, rats treated with peptide-conjugated DOX-loaded liposome formulations exhibited significantly suppressed tumor growth. Out of all the treatments, ${ }^{186}$ Re-BMEDA solution resulted in the highest mean tumor volume of $500 \mathrm{~mm}^{3}$.

Throughout the study, a constant tumor volume was maintained by treatment with the peptide-conjugated DOX-loaded liposome formulation and a mean of almost $90 \%$ tumor regression was observed in this group. This clearly indicates the high efficacy of peptide-conjugated DOX-loaded liposome for controlling the spread of cancer metastasis. When tumor volumes were normalized, the peptide-conjugated DOX-loaded liposome formulation showed less than a 3 times enhancement in the overall bulk of tumor, which was far less than for the control group in which the final bulk increased 20 times. The efficacy of antitumor activity was found to be in the following order: peptide-conjugated DOXloaded liposomes $>$ unlabeled liposomes $>186$ Re-perrhenate solution $>{ }^{186}$ Re-BMEDA solution. 


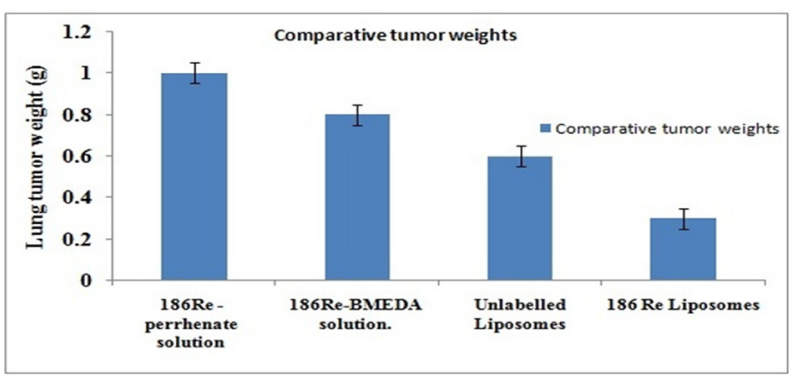

Figure 5. Comparison of antitumor efficacy in terms of tumor weight of ${ }^{186}$ Re-liposomes, unlabelled liposomes, ${ }^{186}$ Re-perrhenate solution and ${ }^{186}$ Re-BMEDA solution determined in SCC cell lines introduced to $B A L B / C-n u / n u$ athymic nude rats.

With respect to the tumor weights, the peptideconjugated DOX-loaded liposome formulation resulted in the smallest tumors $(0.3 \mathrm{~g})$ followed by the control group $(1.0 \mathrm{~g})$ as shown in Fig. 5. The maximal antitumor activity of the peptide-conjugated DOX-loaded liposome formulation was due to its ability to bind to the overexpressed receptors on the cells, leading to remarkably higher drug concentrations and accumulation within cancerous tissues. The median survival of the animals treated with the peptide-conjugated DOX-loaded liposome formulation was found to be $100 \%$.

\section{CONCLUSION}

The present investigation showed that peptide-conjugated DOX-loaded liposomes significantly suppressed the tumor growth in a nude rat model for human head and neck cancer. These results suggest the significant potential of liposomes as a carrier for clinical applications in head and neck cancer.

\section{Ethical statement}

Herewith we confirm that the authors are accountable for all aspects of the work (if applied, including full data access, the integrity of the data and the accuracy of the data analysis) ensuring that questions related to the accuracy or integrity of any part of the work are appropriately investigated and resolved.

\section{REFERENCES}

Bao A, Phillips WT, Goins B (2006) Setup and characterization of a human head and neck squamous cell carcinoma xenograft model in nude rats. Otolaryngol Head Neck Surg. 135: 853-857. https://doi. org/10.1016/j.otohns.2006.06.1257

Cheng L, Huang FZ, Cheng LF, Zhu YQ, Hu Q, Li L, Wei L, Chen DW (2014) GE11-modified liposomes for non-small cell lung cancer targeting: preparation, ex vitro and in vivo evaluation. Int. J. Nanomedicine 9: 921-935. https://doi.org/10.2147/IJN.S53310

Chung CH, Parker JS, Karaca G (2004) Molecular classification of head and neck squamous cell carcinomas using patterns of gene expression. Cancer Cell. 5: 489-500. https://doi.org/10.1016/s15356108(04)00112-6

Cohen EE, Haraf DJ, Kunnavakkam R (2010) Epidermal growth factor receptor inhibitor gefitinib added to chemoradiotherapy in locally advanced head and neck cancer. J. Clin. Oncol. 28: 3336-3343. https://doi.org/10.1200/JCO.2009.27.0397

Dietz A, Flentje M, Hagen R (2010) Docetaxel, cisplatin (TP), and radiation with or without cetuximab in advanced larynx carcinoma (DeLOS II trial). J. Clin. Oncol. 28: 15s (Suppl.): TPS265. https:// doi.org/10.1093/annonc/mdy332

Elser C, Siu LL, Winquist E (2007) Phase II trial of sorafenib in patients with recurrent or metastatic squamous cell carcinoma of the head and neck or nasopharyngeal carcinoma. J. Clin. Onco. 25: 37663773. https://doi.org/10.1200/JCO.2006.10.2871

Farokhzad OC, Langer R (2009) Impact of nanotechnology on drug delivery. ACS Nano 3: 16-20. https://doi.org/10.1021/nn900002m

Haddad RI, Tishler RB, Norris C (2009) Phase I study of C-TPF in patients with locally advanced squamous cell carcinoma of the head and neck. J. Clin. Oncol. 27: 4448-4453. https://doi.org/10.1200/ JCO.2009.22.1333

Harrington KJ, Mohammadtaghi S, Uster PS (2001) Effective targeting of solid tumours in patients with locally advanced cancers by radiolabelled pegylated liposomes. Clin. Cancer Res. 7: 243-254. https:// doi.org/20.1046/clcr.5-2020500

Head and Neck. Cancers. NCI. March 29, 2017. Retrieved 17 September 2017.

Herbst RS, Arquette M, Shin DM (2005) Phase II multicenter study of the epidermal growth factor receptor antibody cetuximab and cisplatin for recurrent and refractory squamous cell carcinoma of the head and neck. J. Clin. Oncol. 23: 5578-5587. https://doi. org/10.1200/JCO.2005.07.120

https://en.wikipedia.org/wiki/Head_and_neck_cancer

Poon RT, Fan ST, Tsang FH, Wong J (2002) Locoregional therapies for hepatocellular carcinoma: a critical review from the surgeon's perspective. Ann. Surg. 235: 466-486. https://doi. org/10.1097/00000658-200204000-00004

Qian J, Fen GS, Vogl T (2003) Combined interventional therapies of hepatocellular carcinoma: Review. World J. Gastroenterol. 9: 1885 1891. https://doi.org/10.3748/wjg.v9.19.1885

Raghavan R, Brady ML, Rodriguez-Ponce MI, Hartlep A, Pedain C, Sampson JH (2006) Convection enhanced delivery of therapeutics for brain disease, and its optimization. Neurosurg. Focus 20: E12,113. https://doi.org/10.1155/2019/9342796

World Cancer Report 2014. World Health Organization. 2014. pp. Chapter 5.8 . 